\title{
Prophage exotoxins enhance colonization fitness in epidemic scarlet fever-causing Streptococcus pyogenes
}

Stephan Brouwer ${ }^{1,15}$, Timothy C. Barnett (1) 1,2,15, Diane Ly ${ }^{3,4}$, Katherine J. Kasper ${ }^{5}$, David M. P. De Oliveira (iD ${ }^{1}$, Tania Rivera-Hernandez', Amanda J. Cork', Liam Mclntyre ${ }^{6}$, Magnus G. Jespersen (1) ${ }^{6}$, Johanna Richter ${ }^{1}$, Benjamin L. Schulz', Gordon Dougan 7,8, Victor Nizet (1) 9, Kwok-Yung Yuen (10) 10,11,12, Yuanhai You ${ }^{13}$, John K. McCormick (1D 5,14,16, Martina L. Sanderson-Smith (10) ${ }^{3,4,16}$, Mark R. Davies (D) ${ }^{1,6,16}$ \&

Mark J. Walker (1) 1,16凶

The re-emergence of scarlet fever poses a new global public health threat. The capacity of North-East Asian serotype M12 (emm12) Streptococcus pyogenes (group A Streptococcus, GAS) to cause scarlet fever has been linked epidemiologically to the presence of novel prophages, including prophage $\Phi H K U$.vir encoding the secreted superantigens SSA and SpeC and the DNase Spd1. Here, we report the molecular characterization of $\Phi H K U$.vir-encoded exotoxins. We demonstrate that streptolysin O (SLO)-induced glutathione efflux from host cellular stores is a previously unappreciated GAS virulence mechanism that promotes SSA release and activity, representing the first description of a thiol-activated bacterial superantigen. Spd1 is required for resistance to neutrophil killing. Investigating single, double and triple isogenic knockout mutants of the $\Phi H K U$.vir-encoded exotoxins, we find that SpeC and Spd1 act synergistically to facilitate nasopharyngeal colonization in a mouse model. These results offer insight into the pathogenesis of scarlet fever-causing GAS mediated by prophage $\Phi H K U$.vir exotoxins.

\footnotetext{
${ }^{1}$ Australian Infectious Diseases Research Centre and School of Chemistry and Molecular Biosciences, The University of Queensland, St. Lucia, QLD, Australia. ${ }^{2}$ Wesfarmers Centre for Vaccines and Infectious Diseases, Telethon Kids Institute, University of Western Australia, Nedlands, WA, Australia. ${ }^{3}$ Illawarra Health and Medical Research Institute, Wollongong, NSW, Australia. ${ }^{4}$ Molecular Horizons and School of Chemistry and Molecular Bioscience, University of Wollongong, Wollongong, NSW, Australia. ${ }^{5}$ Department of Microbiology and Immunology and the Centre for Human Immunology, Schulich School of Medicine \& Dentistry, Western University, London, ON, Canada. ${ }^{6}$ Department of Microbiology and Immunology at the Peter Doherty Institute for Infection and Immunity, The University of Melbourne, Melbourne, VIC, Australia. ${ }^{7}$ The Wellcome Trust Sanger Institute, Hinxton, Cambridge, UK. ${ }^{8}$ Department of Medicine, University of Cambridge, Cambridge, UK. ${ }^{9}$ Department of Pediatrics, University of California San Diego, La Jolla, CA, USA. ${ }^{10}$ State Key Laboratory of Emerging Infectious Diseases, The University of Hong Kong, Pokfulam, Hong Kong Special Administrative Region, Hong Kong, China. ${ }^{11}$ Department of Microbiology, Li Ka Shing Faculty of Medicine, The University of Hong Kong, Pokfulam, Hong Kong Special Administrative Region, Hong Kong, China.

12 Department of Clinical Microbiology and Infection Control, The University of Hong Kong-Shenzhen Hospital, Shenzhen, Guangdong Province, Hong Kong, China. ${ }^{13}$ State Key Laboratory for Infectious Disease Prevention and Control, Collaborative Innovation Centre for Diagnosis and Treatment of Infectious Diseases, National Institute for Communicable Disease Control and Prevention, Chinese Centre for Disease Control and Prevention, Beijing 102206, China. ${ }^{14}$ Lawson Health Research Institute, London, ON, Canada. ${ }^{15}$ These authors contributed equally: Stephan Brouwer, Timothy C. Barnett.

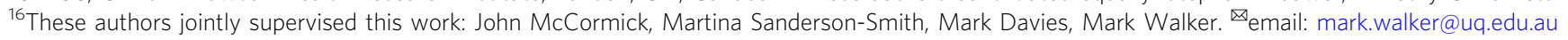


S carlet fever is a superantigen-mediated acute infectious disease caused by the human-adapted pathogen group A Streptococcus (GAS). Scarlet fever was a leading cause of death in children in the early 1900s, but its incidence steadily declined during the 20th century ${ }^{1,2}$. Large regional outbreaks of scarlet fever re-emerged in North-East Asia in 2011, and the United Kingdom in 2014 (refs ${ }^{3-10}$ ), with factors driving disease resurgence remaining a mystery. Alarmingly, recent studies report GAS outbreak strains in other countries ${ }^{11-13}$, heightening the need for global surveillance ${ }^{14}$.

Potential triggers for these new scarlet fever epidemics remain unclear, but accumulating epidemiological evidence indicates that novel prophages and antibiotic resistance elements have played a significant role in the evolution, virulence and diversification of scarlet fever causing GAS strains in North-East Asia ${ }^{4,15-17}$. Detailed phylogenetic analyses of GAS outbreak isolates from mainland China and Hong Kong prove that the increase in scarlet fever cases was neither emm-type specific nor caused by the spread of a single scarlet fever producing clone. Instead, multiclonal scarlet fever outbreak strains are commonly associated with the acquisition of related exotoxin-carrying mobile genetic elements ${ }^{15,17}$. Prophages encoding combinations of the streptococcal superantigens SSA and SpeC, and the DNase Spd1, appear to play an important role in the evolutionary pathway that lead to the emergence of more virulent strains, particularly in North-East Asia ${ }^{4-6,15-18}$. However, robust evidence defining the mechanistic contribution of prophage-encoded exotoxins to the pathogenesis of scarlet fever is lacking.

A universal feature of superantigens is their ability to cross-link major histocompatibility complex II molecules on antigenpresenting cells and the variable region of the $\beta$-chain of T-cell receptor (TCR). This cross-linkage results in TCR V $\beta$-specific activation of large populations of human $\mathrm{T}$ cells, without prior antigen processing, rendering superantigens the most potent $\mathrm{T}$ cell mitogens known to date ${ }^{19}$. Recent studies suggest that such T-cell activation contributes to the establishment of GAS infection at mucosal surfaces ${ }^{20,21}$. Here, we investigate the regulation of $\Phi H K U . v i r$ encoded exotoxin genes ssa, speC and spd1, and their impact on the virulence of scarlet fever-causing GAS. Exotoxin-driven enhanced colonization provides an evidencebased hypothesis for the reemergence of scarlet fever globally.

\section{Results}

Regulation of $\Phi$ HKU.vir exotoxins. The majority of GAS emm 12 clones from scarlet fever outbreaks in North-East Asia carry superantigens SSA and SpeC and the DNase Spd1, as well as integrative and conjugative elements (ICE) encoding tetracycline $($ tet $M)$ and macrolide $(\mathrm{ermB})$ resistance ${ }^{4,15,17}$. Penicillin remains the treatment of choice for GAS pharyngitis. However, in many countries macrolides are commonly used as first-line therapy for upper respiratory tract infections in primary health-care settings ${ }^{22}$. To investigate the possibility that antibiotic treatment stress may influence regulation of prophage-encoded toxins, macrolideresistant GAS emm12 scarlet fever isolate HKU16 harboring ФHKU.vir and ICE-emm 12 was grown in THY medium containing erythromycin $\left(2 \mu \mathrm{g} \mathrm{ml}^{-1}\right)$, the recommended drug in patients with penicillin hypersensitivity ${ }^{23}$. RNA-seq analysis showed that erythromycin treatment did not affect the gene expression pattern of $\Phi$ HKU.vir (Fig. 1a), whereas expression levels of ICE-emm12encoded ermB gene and the adjacent transposase gene were significantly increased (Supplementary Fig. 1). This finding aligns with previous observations that erythromycin induces ermB, facilitating its mRNA stabilization and processing ${ }^{24}$. Mitomycin C, a DNAdamaging agent known to induce GAS prophage ${ }^{25}$, effectively induced $\Phi H K U . v i r$ housekeeping and structural gene expression
(Fig. 1a, Supplementary Fig. 1). Similar to prophage-encoded virulence factor cargo genes in emm $3 \mathrm{GAS}^{25}$, mitomycin C did not induce expression of the virulence cargo genes $s s a, s p e C$ and $s p d 1$, pointing to differential control of exotoxin expression in $\Phi$ HKU.vir.

Thiol-mediated induction of SSA release. Neutralizing antibodies against SSA and SpeC have been detected in human serum $^{26-28}$, suggesting that GAS expresses the two superantigens during infection. While there is further experimental evidence that phage-associated exotoxins $\mathrm{SpeC}$ and $\mathrm{Spd} 1$ are induced during host-pathogen interactions ${ }^{29,30}$, comparatively less is known about the control of SSA expression. The ssa gene is frequently associated with scarlet fever isolates from North-East Asia 15,17 . As SSA production is detectable upon growth in a chemically defined medium ${ }^{16}$, we undertook a limited small molecule screen that identified cysteine as a factor specifically increasing abundance of the exotoxin SSA in culture supernatants (Fig. 1b, Supplementary Fig. 2). Cysteine is uniquely chemically reactive, due to its thiol (or "sulfhydryl") group that is a major target of reactive oxygen species and readily forms disulfide bonds with itself. We therefore examined whether SSA production was subject to thiol-mediated regulation. Both dithiothreitol (DTT) and the reduced form of glutathione (GSH) increased SSA production in GAS culture supernatants (Fig. 1b). By contrast, oxidized glutathione (GSSG) did not enhance SSA levels. Higher SSA production was also detected in GAS cultures treated with thiolfree reducing agent tris(2-carboxyethyl)phosphine (TCEP), suggesting that exposure to reducing conditions enhances SSA production. The levels of secreted SpeC and Spd1 were unaffected by any of these treatments (Fig. 1b). Quantitative real-time polymerase chain reaction (PCR) showed no increase in abundance of the ssa and speC transcripts, suggesting that reducing agents act as post-transcriptional enhancers of SSA release (Fig. 1c). To validate the requirement for thiols (reducing conditions) in SSA regulation, we also performed alkylation of cysteine with acrylamide prior to treatment to irreversibly block the free thiol group, resulting in a clear reduction of SSA, but not SpeC, release (Supplementary Fig. 3a).

SSA is a thiol-activated superantigen. SSA contains a surfaceexposed Cys-26 residue that, based on the crystal structure of the homologous SpeA superantigen in complex with TCR V $\beta^{31}$, is predicted to lie within the TCR binding interface (Supplementary Fig. 3b). Prior site-directed mutagenesis has revealed a role for Cys-26 in the mitogenic activity of SSA on human T cells by preventing disulphide-linked dimer formation between the surface-exposed Cys-26 residues of SSA ${ }^{32}$. Although a SSA dimer was not detectable in HKU16 culture supernatants (Supplementary Fig. 3c), possibly due to very low concentration levels preventing dimerization as previously observed for other streptococcal superantigens ${ }^{33,34}$, we detected dimer formation by purified recombinant SSA (Supplementary Fig. 3d) which led us to investigate possible redox sensitivity of SSA activity. GSH, the major low-molecular-weight thiol in living cells, markedly increased the mitogenic potency of recombinant SSA on human $\mathrm{T}$ cells by $\sim 10$-fold as assessed by enhanced IL- 2 production (Fig. 1d). However, thiol activation by GSH was absent in SSA carrying a cysteine-to-serine substitution at position $26\left(\mathrm{SSA}_{\mathrm{C} 26 \mathrm{~S}}\right)$, underscoring a critical role for the Cys-26 residue in thiolmediated activation. In contrast to SSA, the activity of SpeC, one of the most potent T-cell mitogens ${ }^{35}$, was unaffected by GSH treatment (Fig. 1d). These data establish a unique role for thiols in SSA regulation and support a model where reducing agents not only increase levels of extracellular SSA monomer, but also directly enhance SSA-mediated $\mathrm{T}$ cell stimulation. To our 
a

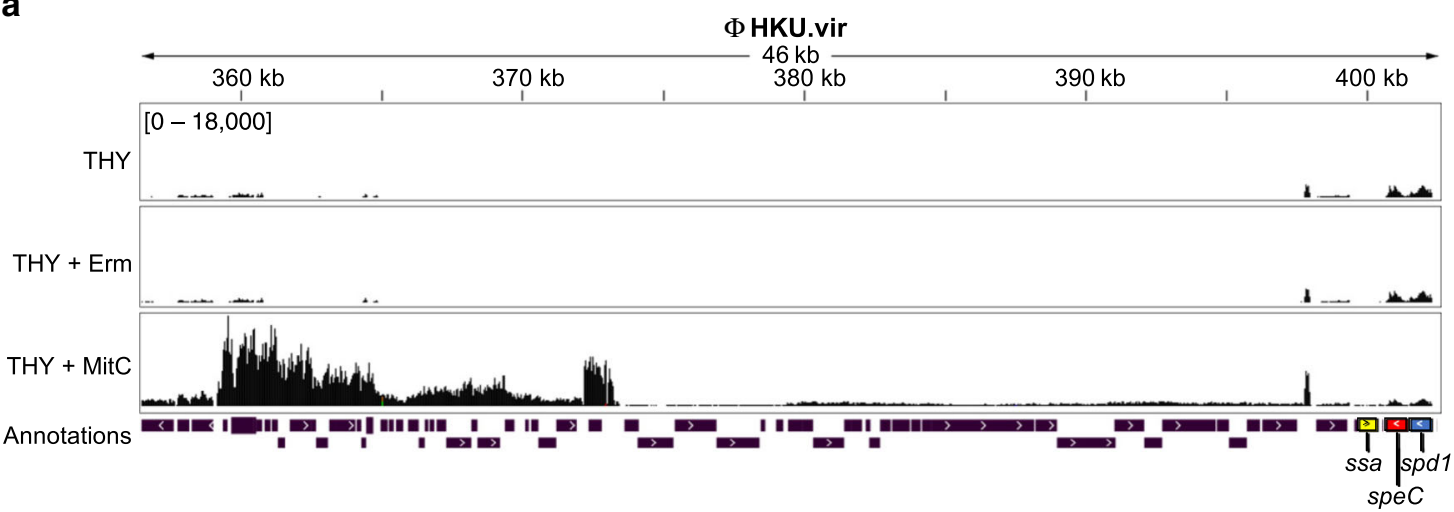

b
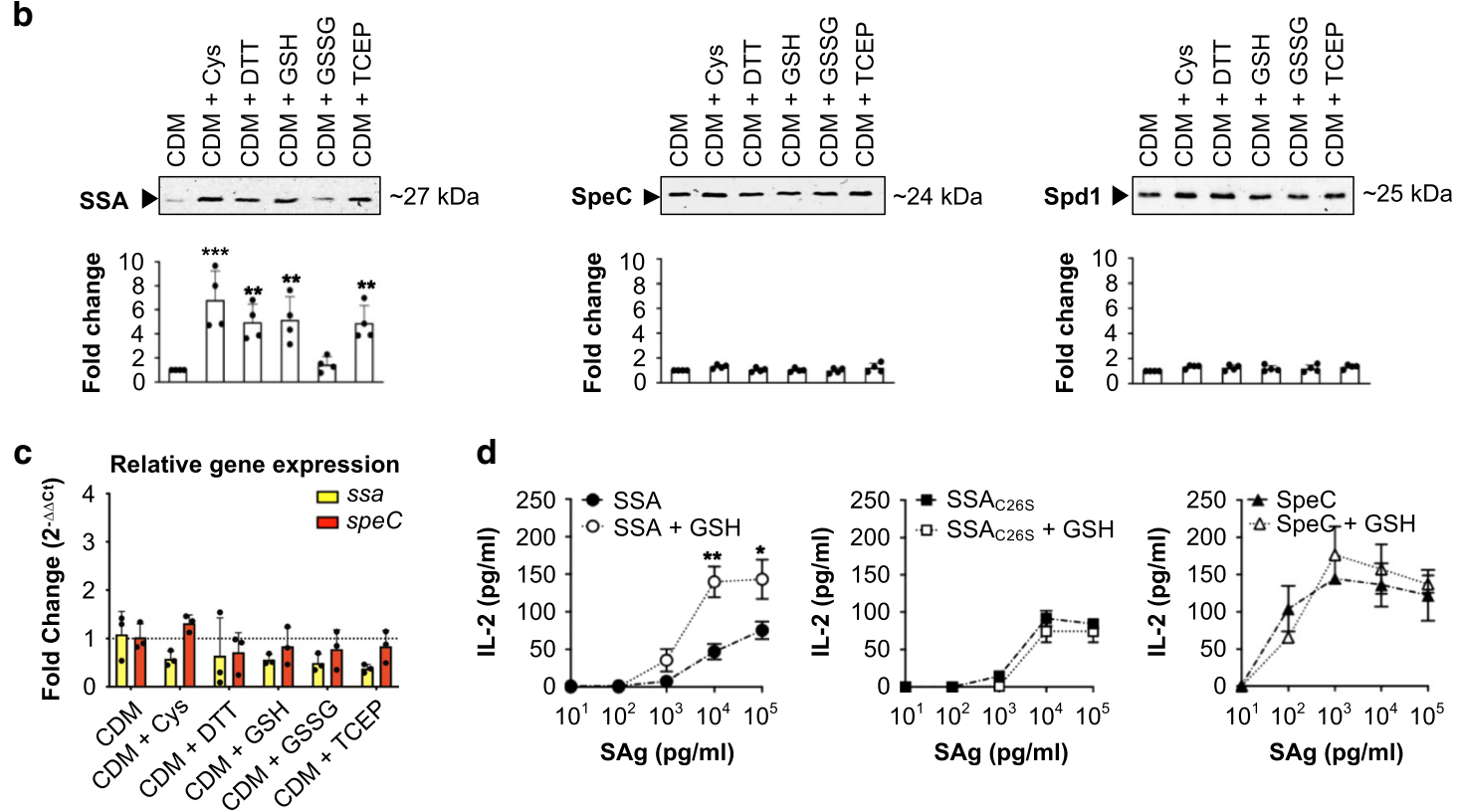

Fig. 1 Post-transcriptional thiol-based regulation of SSA. a RNAseq expression profile of $\Phi H K U . v i r$ in the macrolide- and tetracycline-resistant GAS emm12 isolate HKU16, grown in THY broth with sub-inhibitory concentrations of erythromycin (Erm) and mitomycin C (MitC). The plots illustrate the overall coverage distribution displaying the total number of sequenced reads. The region that encodes exotoxin genes (ssa in yellow, speC in red, and spd 1 in blue) is indicated. $\mathbf{b}$ Immunoblot detection of SSA, SpeC, and Spd1 in culture supernatants of HKU16 grown in a chemically defined medium (CDM) in the presence of various redox-active compounds. Western blot signal intensities were quantified with ImageJ. Data are presented as mean values \pm SD. Statistical significance was assessed using one-way ANOVA with Dunnett's multiple comparisons post hoc test against the CDM control group ( ${ }^{\star \star \star} p<$ 0.001 for $\mathrm{CDM}+\mathrm{Cys},{ }^{\star \star} p=0.008$ for CDM + DTT, ${ }^{\star \star} p=0.006$ for CDM + GSH, and ${ }^{\star \star} p=0.01$ for CDM + TCEP) $(n=4)$. c Quantitative real-time PCR of ssa and speC transcripts in HKU16 grown in CDM treated with $2 \mathrm{mM}$ of the indicated redox-active compounds. Data from three biological replicates are presented as mean values \pm SD. d Superantigen (SAg) activation of human T cells with SSA (circular), SSA $\mathrm{C}_{26 \mathrm{~S}}$ (square), and SpeC (triangular) at the indicated concentrations in absence (black; dash-dot line) or presence of $2 \mathrm{mM}$ of GSH (white; dotted line), using human IL-2 as a readout. Results are expressed as the mean \pm SEM from three independent experiments from one representative donor (out of three independent donors). Statistical significance was assessed by two-tailed unpaired Student's $t$ test ( ${ }^{\star \star} p=0.0062$ for SSA + GSH at a Sag concentration of $10^{4} \mathrm{pgmL}^{-1}$, and ${ }^{\star} p=0.0306$ for $\mathrm{SSA}+\mathrm{GSH}$ at a Sag concentration of $10^{5} \mathrm{pg} \mathrm{mL}^{-1}$ ). Source data are provided as a Source Data file.

knowledge, this is the first report of a thiol-activated superantigen.

Streptolysin O mediates release of host GSH. Like other species of pathogenic Gram-positive bacteria, GAS produces a cholesterol-dependent cytolysin, streptolysin O (SLO), that perforates host cell membranes ${ }^{36}$. In contrast to plasma and other extracellular fluids that are low in thiol-based antioxidants, the cytosol of mammalian cells is a highly reducing compartment where thiols are present at high concentration. The most abundant non-protein thiol in mammalian cells is GSH, with intracellular concentrations typically in the millimolar range $(\sim 1-11$ $\mathrm{mM})$, compared to extracellular concentrations in the low micromolar range ${ }^{37}$. This GSH concentration differential across the plasma membrane led us to speculate that host cell membrane damage by SLO, itself subject to thiol activation ${ }^{38}$, could provide extracellular GAS with access to the intracellular GSH pool, altering the redox environment and supporting SSA activation.

To test this hypothesis, we first quantified GSH release after treatment of whole human blood with increasing concentrations of purified SLO. SLO lysed red blood cells (Fig. 2a), and both hemoglobin and total GSH (GSH + GSSG) accumulated rapidly in plasma in a dose-dependent manner (Fig. 2a). In the context of live GAS, wild-type scarlet fever-associated strain HKU16 caused significant red blood cell hemolysis after $4 \mathrm{~h}$ growth in human blood (Fig. 2b), paralleled by a significant and substantial release of GSH into plasma (Fig. 2c). By contrast, an isogenic GAS 

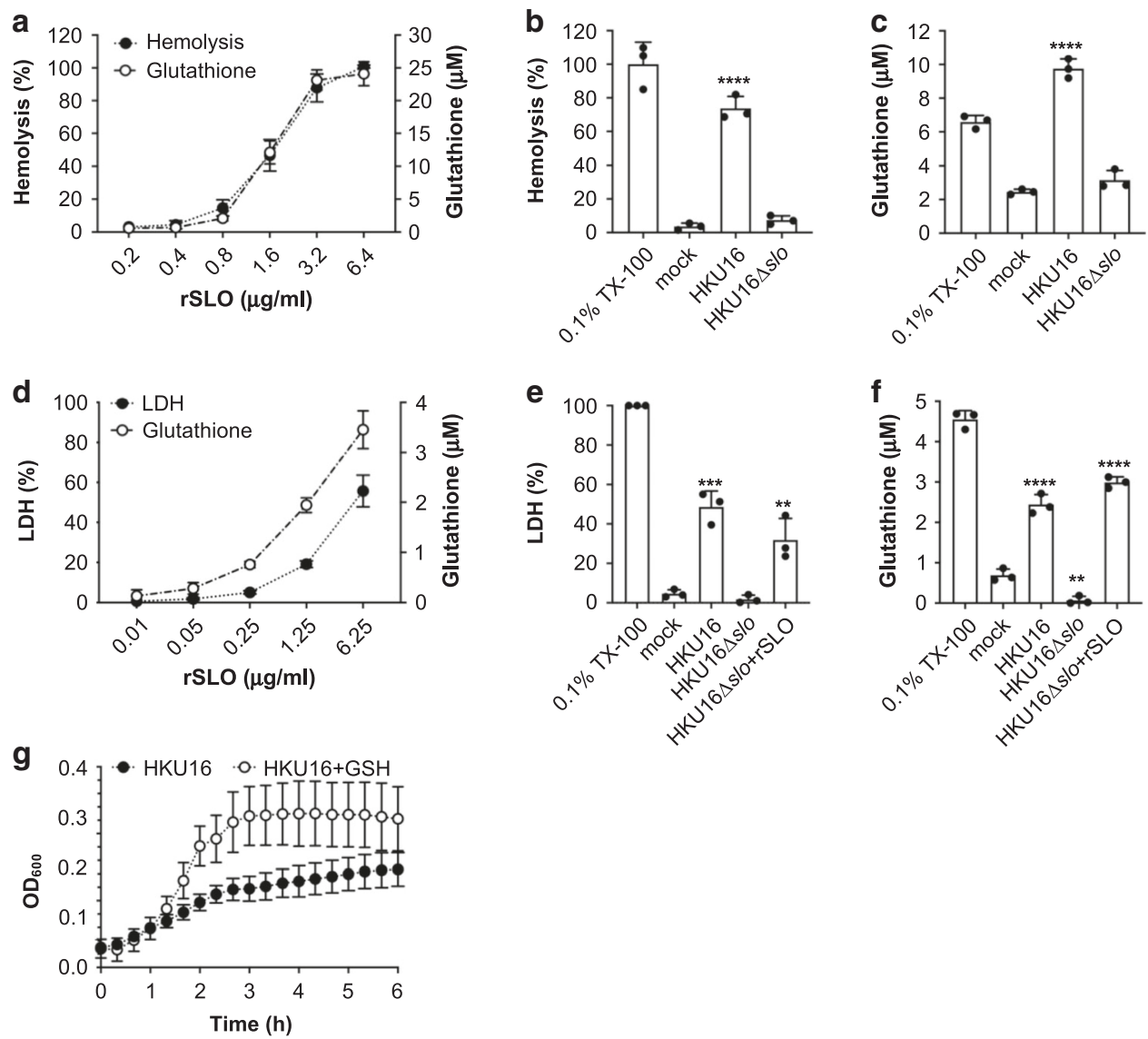

Fig. 2 The cytotoxic activity of SLO causes the release of host cytosolic glutathione. a Dose-dependent hemolytic activity of purified recombinant SLO (rSLO) in whole human blood is accompanied by an extracellular accumulation of glutathione. Hemolysis is expressed as percentage \pm SD with respect to the positive control (cells treated with $0.1 \%$ Triton X-100 (TX-100) $(n=4)$. b Hemolytic activity of indicated HKU16 strains is expressed as percentage \pm SD $(n=3)$ with respect to the positive control (cells treated with $0.1 \%$ TX-100). Blood treated with HBSS (mock) served as a negative control. Statistical

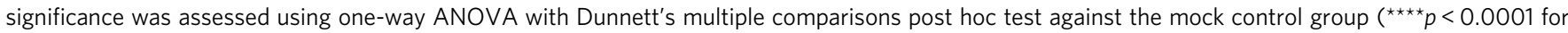
HKU16). c Extracellular accumulation of glutathione in blood $(n=3)$ infected with indicated HKU16 strains. Blood treated with HBSS (mock) served as a negative control. Statistical significance was assessed using one-way ANOVA with Dunnett's multiple comparisons post hoc test against the mock control group ( ${ }^{\star \star \star \star} p<0.0001$ for HKU16). d Release of lactate dehydrogenase (LDH) (closed circles) and glutathione (open circles) by pharyngeal cells treated with varying concentrations of recombinant SLO. The release of $\mathrm{LDH}$ and glutathione into the culture medium was assessed after $2 \mathrm{~h}$ of treatment. LDH release is expressed as percentage \pm SD with respect to the positive control (cells treated with $0.2 \%$ TX-100). Cells treated with growth medium (mock) served as a negative control. Data shown are representative of three independent experiments. LDH (e) and glutathione (f) release by pharyngeal cells challenged with indicated HKU16 strains at a multiplicity of infection of 20:1 (bacterial colony forming units (CFU):cells), assessed at $2 \mathrm{~h}$ post infection $(n=3)$. Where indicated, rSLO was added to HKU16 $\Delta$ slo-infected cells at a concentration of $6.25 \mu \mathrm{g} / \mathrm{ml}$. Statistical significance was assessed using oneway ANOVA with Dunnett's multiple comparisons post hoc test against the mock control group ( $\mathbf{e}^{\star \star \star} p=0.0002$ for HKU16, and ${ }^{\star \star} p=0.0034$ for

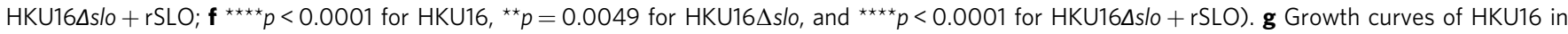
pharyngeal cell-free culture medium (EMEM $+10 \% \mathrm{FBS}$ ) supplemented with $2 \mathrm{mM}$ of GSH $(n=3)$. All data are presented as mean values \pm SD. Source data are provided as a Source Data file.

HKU16 $\Delta$ slo mutant did not induce hemolysis and plasma levels of GSH were unchanged (Fig. 2b, c).

GAS serotype M12 strains belong to $\mathrm{emm}$ pattern A-C and have been designated as "throat specialists" (ref. ${ }^{39}$ ). In this context, we used human pharyngeal cells (Detroit 562) to study the effect of SLO-induced pore formation on GSH release as a pharyngitis-relevant cellular model. Lactate-dehydrogenase $(\mathrm{LDH})$ release into the media serves as a marker of host cell membrane integrity. As expected, SLO caused a dose-dependent release of $\mathrm{LDH}$ of $\sim 50 \%$ at $6.25 \mu \mathrm{g} \mathrm{ml}^{-1}$, confirming disruption of the cell membrane structure (Fig. 2d). Dose-dependent cell membrane damage following SLO exposure was again associated with a progressive increase in GSH level in the media (Fig. 2d), indicating that SLO-induced membrane disruption was sufficient to trigger extracellular release of host cytosolic GSH stores.
Next, levels of LDH and GSH released by pharyngeal cells following infection with live GAS (multiplicity of infection $=20$ bacterial colony forming units (CFUs):cell) were measured. At $2 \mathrm{~h}$ post infection, wild-type GAS HKU16 but not the HKU16 $\Delta$ slo mutant induced a significant increase in levels of secreted LDH and GSH (Fig. 2e, f). The addition of purified pore-forming protein toxin SLO $\left(6.25 \mu \mathrm{g} \mathrm{ml}^{-1}\right)$ to pharyngeal cells grown in the presence of HKU16 $\Delta$ slo markedly elevated extracellular LDH and GSH to wild-type HKU16 levels during co-culture. To examine whether the lack of GSH release following infection with HKU16 $\Delta$ slo (Fig. 2f) might impact other aspects of GAS biology, we measured growth in cell-free medium with and without GSH supplementation. Supplementation with GSH strongly promoted growth of wildtype GAS HKU16 in cell-free medium (Fig. 2g), showing that host-derived GSH is utilized by GAS for other 
physiological pathways. Taken together, our data demonstrate that SLO is highly effective at triggering the release of significant amounts of GSH from host cells, which is utilized for extracellular growth of GAS and likely provides a reducing extracellular microenvironment required for efficient SSA activation in vivo.

DNase Spd1 enhances HKU16 resistance to neutrophil killing. Horizontal transmission of bacteriophage encoding DNase Sda1/ SdaD2 has played a critical role in the emergence and global dissemination of the highly virulent M1T1 clone ${ }^{40-42}$. The phageencoded DNase Spd1 is linked with the expansion of scarlet fever GAS in North-East Asia ${ }^{15}$. In contrast to Sda1 (ref. ${ }^{42}$ ), which is also carried by $>95 \%$ of the global GAS emm12 population including HKU16 (refs ${ }^{12,15}$ ), the contribution of Spd1 to GAS pathogenesis remains largely unexplored, although this nuclease has previously been shown to play a role in nasal shedding in emm3 GAS 43 .

Unlike the knockout strains HKU16 1 ssa and HKU16 $\Delta$ speC, DNase knockout strain HKU16 spd1 showed significantly attenuated growth in human blood (Fig. 3a). Reinforcing these results, complementation of HKU16 $\Delta$ spd1 with the wild-type spdl gene (HKU16 $\left.\Delta p d 1^{++}\right)$restored growth in human blood (Fig. 3a). Neutrophils are the first immune cell responders to sites of bacterial infection, and thus play a critical role in controlling GAS infection. Examining the role of Spd1 in bacterial susceptibility to human neutrophil killing, knockout strain HKU16 $\Delta$ spd1 showed significantly reduced survival compared to the wild-type and complemented HKU16 strains (Fig. 3b). Formation of web-like lattices composed of chromatin and granular proteins, known as neutrophil extracellular traps (NETs), is a well-established antimicrobial mechanism ${ }^{44}$. Multiple pathogenic microorganisms, including GAS, secrete DNases that dissolve NETs and allow escape from neutrophil mediated killing ${ }^{45}$. To determine the ability of Spd1 to facilitate NET degradation, we used phorbol-myristate acetate (PMA) to induce high levels of NETs from freshly isolated human neutrophils (Fig. 3c) that are sensitive to bovine pancreatic DNase I (Fig. 3c, d). We then incubated PMA-stimulated neutrophils with GFPexpressing GAS for $30 \mathrm{~min}$. NETs exposed to HKU16 $\Delta$ spd1 remained intact and covered a significantly greater area in the absence of Spd1 $(64.1 \pm 3.3 \%)$ compared to NETs infected with wildtype HKU16 $(24.5 \pm 4.1 \%)$ and HKU16 $\Delta s p d 1^{++}(21.9 \pm$ $5.2 \%$ ) (Fig. 3e, f). Similar levels of NET degradation were displayed by wildtype HKU16 and HKU16 spd1 ${ }^{++}$(Fig. 3e, f). These findings demonstrate that Spd1 promotes growth of HKU16 in whole blood, reduces susceptibility to neutrophil mediated killing and facilitates NET degradation.

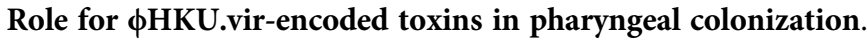
Previous studies have shown that intranasal infection of mice with GAS can serve as a model to study pharyngeal infection in humans $\mathrm{S}^{20,21,46,47}$. Humanized mice that express HLA-DR4 and HLA-DQ8 are susceptible to acute nasopharyngeal infection by SpeA-carrying emm 18 GAS $^{20,21}$. To evaluate the role of HKU16 exotoxins in nasopharyngeal infection, we investigated the ability of wild-type and isogenic mutants to colonize the nasopharynx of HLA-B6 mice transgenic for human CD4 and HLA-DR4-DQ8 genes $^{48}$. The in vitro growth phenotype of all single, double and triple HKU16 isogenic knockout mutants (Fig. 4a) was indistinguishable from the parental strain (Fig. $4 \mathrm{~b}$ ) and all mutant strains were defective for production of the targeted exotoxins SSA, SpeC, Spd1, and SLO (Fig. 4c). HLA-B6 mice were infected intranasally with wild-type HKU16 or isogenic mutants. At $48 \mathrm{~h}$ post infection, significantly fewer bacterial CFUs were recovered from the complete nasal turbinates of mice infected with
HKU16 speC/spd1 compared to wild-type HKU16 (Fig. 4d). Single isogenic mutant strains of $\Phi$ HKU.vir-encoded exotoxins did not show reduced colonization efficiency suggesting that SpeC and Spd1 act synergistically to enhance nasopharyngeal infection, nor did the additional knockout of the ssa gene in the triple mutant strain HKU16 $s s a / s p e C / s p d 1$ further reduce colonization. The attenuated virulence phenotype of HKU16 $\Delta s a / s p e C / s p d 1$ could be fully restored by genetic complementation with wildtype ssa, speC, and spd1 genes (HKU16 $\mathrm{ssa} / \mathrm{speC} / \mathrm{spd1} 1^{++}$) (Fig. 4d). Significantly fewer bacterial CFUs were also recovered from HKU16 $\Delta$ slo infected mice (Fig. 4d), confirming the importance of SLO for GAS pathogenicity as demonstrated in previous studies ${ }^{49-51}$. No additional virulence loss was observed upon combining SLO and $\Phi$ HKU.vir-encoded exotoxin mutations (Supplementary Fig. 5).

\section{Discussion}

Mainland China and Hong Kong have witnessed an ongoing outbreak of scarlet fever with $\sim 500,000$ reported cases since 2011 ( $r e f s$ 4,14,15,17,52-55). Alarmingly, case numbers have again significantly increased in recent years posing a heightened global threat to public health ${ }^{12}$ (Supplementary Fig. 4). Previous epidemiological surveillance studies have shown that emm12 is the most prevalent GAS emm genotype in clinical cases of scarlet fever in this region ${ }^{4,15,17}$. In contrast with the United Kingdom epidemic, the expansion of scarlet fever-associated emm12 lineages in North-East Asia has been directly linked to acquisition of two genetic elements: the tet $M$ - and ermB-carrying multidrug resistance element ICE-emm 12 (and its derivatives) and the prophage $\Phi$ HKU.vir, encoding SSA, SpeC and the DNase Spd1 (refs 4,15,56). Consistent with these prior studies, the results presented here demonstrate a direct contribution of $\Phi H K U . v i r$ acquisition to virulence phenotypes of the scarlet fever-causing emm12 reference strain HKU16. Using defined genetic knockouts, our data suggest that SpeC and the DNase Spd1 function synergistically to mediate nasopharyngeal colonization, offering an explanation as to why these genes form a conserved genetic module in a variety of distinct GAS prophage ${ }^{30}$.

We also present new insight into the activation of the scarlet fever-associated superantigen SSA, which we reveal as a thiolactivated superantigen. By providing a mechanistic framework of how extracellular GAS gains access to highly abundant intracellular GSH in vivo, we highlight the relationship between SLOmediated membrane disruption and SSA activity (Fig. 5). Data presented here extend previous studies showing that epithelial cell damage by SLO augments superantigen penetration, which allows for better interaction of superantigens with antigen presenting cells in underlying tissues ${ }^{57}$. Together, these studies suggest that SLO pore formation promotes SSA activation, which may be an important driver in diseases associated with superantigen production, including scarlet fever.

GAS encodes several GSH-dependent proteins, yet the bacterium lacks genes for de novo GSH synthesis. This paradox raises the possibility that GAS may coordinate a range of virulence factors through SLO-mediated GSH release. One such factor is GSH peroxidase $(\mathrm{GpoA})^{58}$, which plays a role in the adaptation of GAS to oxidative stress during inflammation following systemic infection ${ }^{59}$. Microbial acquisition of nutrients in vivo is a fundamental aspect of infectious disease pathogenesis, and intracellular bacterial pathogens capitalize on the ubiquitous and highly abundant cytosolic antioxidant $\mathrm{GSH}^{60,61}$. Our data support a hypothesis in which extracellular bacterial pathogens such as GAS may have evolved a mechanism to target and hijack host cytosolic GSH, consistent with the absence of GSH biosynthetic genes in the GAS genome. While a precise role for SSA in 
a

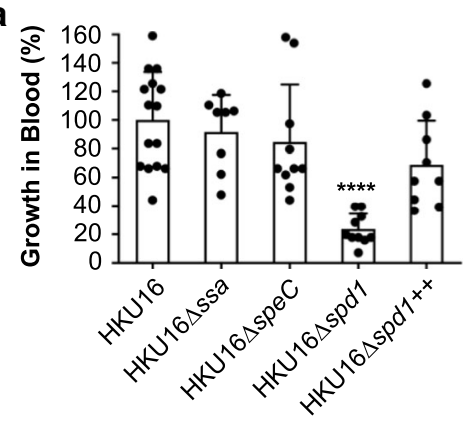

d

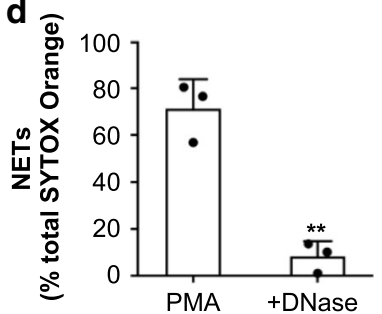

$\mathbf{f}$

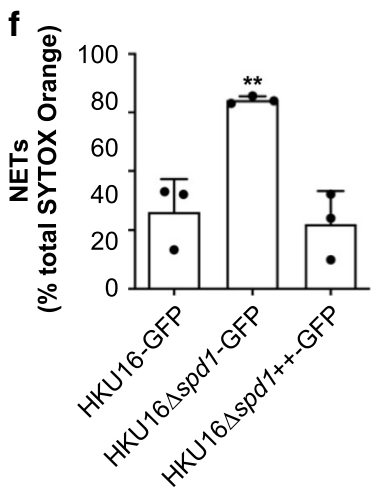

b
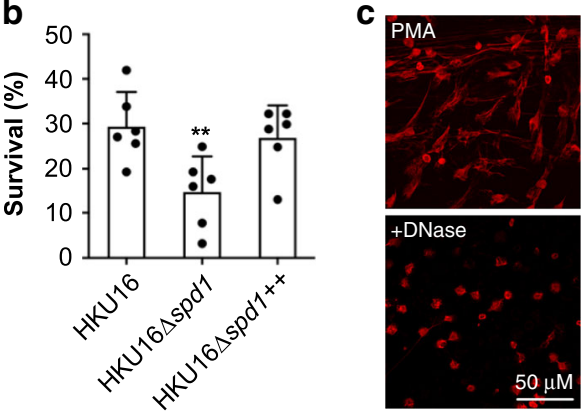

e

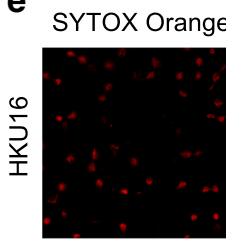

GFP

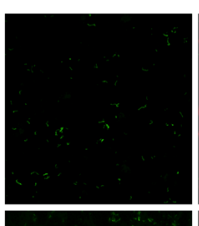

Merge

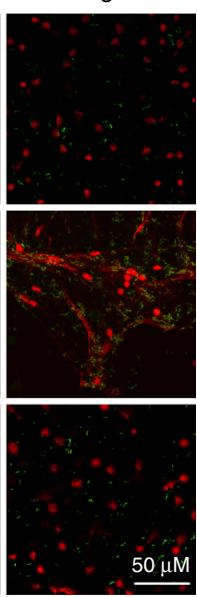

Fig. 3 The $\Phi H K U . v i r-e n c o d e d ~ D N a s e ~ S p d 1$ promotes resistance to neutrophil killing. a Growth of indicated HKU16 strains in whole human blood. Statistical significance was assessed using one-way ANOVA with Dunnett's multiple comparisons post hoc test against the HKU16 control group ( ${ }^{\star \star \star \star} p<$ 0.0001 for HKU16 $\Delta$ spd1). b Human neutrophil killing assay. The data represent the mean \pm SEM of six independent experiments. Statistical significance was assessed using one-way ANOVA with Dunnett's multiple comparisons post hoc test against the HKU16 control group (** $p=0.0093$ for HKU16 $\Delta$ spd1). c Purified human neutrophils were stimulated with $25 \mathrm{nM}$ PMA for $3 \mathrm{~h}$ to induce neutrophil extracellular traps (NETs). NETs were detected using the extracellular DNA stain SYTOX Orange (red) and images captured using confocal microscopy. Panels show formation of NETs (left) and NET degradation following incubation with bovine pancreatic DNase I as a positive control (right). d NET quantification of PMA-stimulated neutrophils in the absence or presence of DNase I. Statistical significance was assessed by two-tailed unpaired Student's $t$ test $\left({ }^{\star \star} p=0.0016\right.$ for DNase treatment).

e Representative images of PMA-stimulated neutrophils following infection with GFP fluorescent GAS (green) for 30 min at a multiplicity of infection of 10 (bacterial CFU:neutrophil). Scale bars represent $50 \mu \mathrm{m}$. f NET quantification of PMA-stimulated neutrophils following incubation with GAS. NET quantification is expressed as a percentage of total SYTOX Orange stained area calculated from a minimum of five randomly selected microscopic fields. Error bars represent the mean \pm SEM from three independent experiments. Statistical significance was assessed using one-way ANOVA with Dunnett's multiple comparisons post hoc test against the HKU16-GFP control group ( ${ }^{\star \star} p=0.0041$ for HKU16 $\Delta$ spd1-GFP). Source data are provided as a Source Data file.

virulence was not conclusively established in the HLA-B6 mouse model, this work exemplifies an interconnected action of GAS virulence determinants such as SLO and SSA, opening new avenues to understand the evolution and emergence of pathogenic clones. As multiple bacterial pathogens encode functional homologs of $\mathrm{SLO}^{36}$, GSH release by cholesterol-dependent cytolysins may constitute a generalized mechanism used by pathogenic bacteria to modulate their physiological response to host cells, including through the post-transcriptional activation of virulence-associated proteins.

Our findings show that GAS HKU16 requires the $\Phi$ HKU.virencoded exotoxins SpeC and Spd1, and SLO, to efficiently colonize the HLA-B6 mouse model. We hypothesize that prophage-encoded exotoxin acquisition has enhanced colonization fitness of scarlet fever-causing GAS emm12 clones in North-East Asia. The atypical presence of genes encoding superantigens such as SSA in emm12 isolates ${ }^{62}$ could provide a framework allowing for clonal expansion of GAS in a naïve population. The spread of such prophage-containing GAS is therefore of great public health concern and heightened efforts are needed to instigate global surveillance systems. Recent evidence of interspecies transfer of speC- and spd1-containing prophage in the United States should serve as a warning for the dissemination of these virulenceenhancing genes into other pathogenic streptococci ${ }^{63}$.

\section{Methods}

Bacterial strains, growth conditions, and mutant construction. The emm 12 GAS scarlet fever isolate HKU16 (ref. ${ }^{4}$ ) and isogenic derivatives were routinely 
a
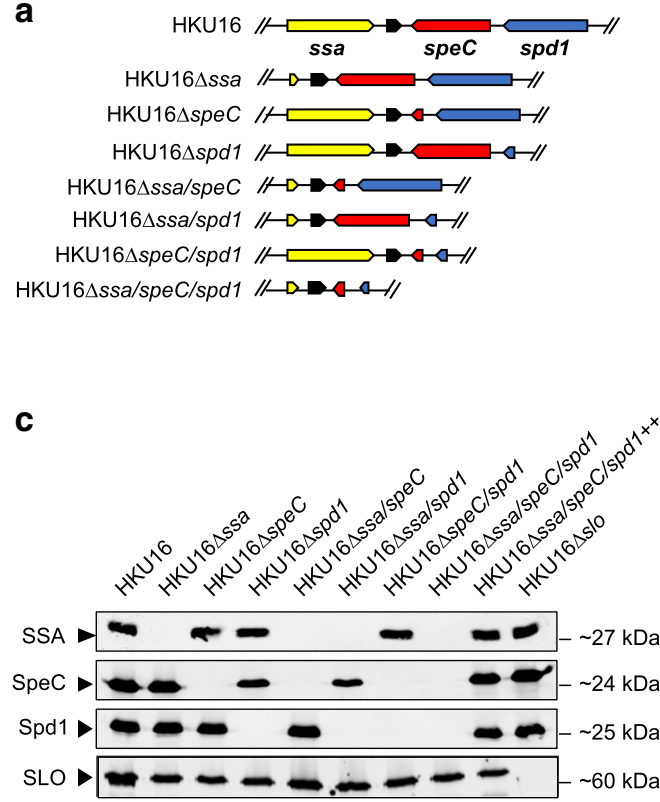

b

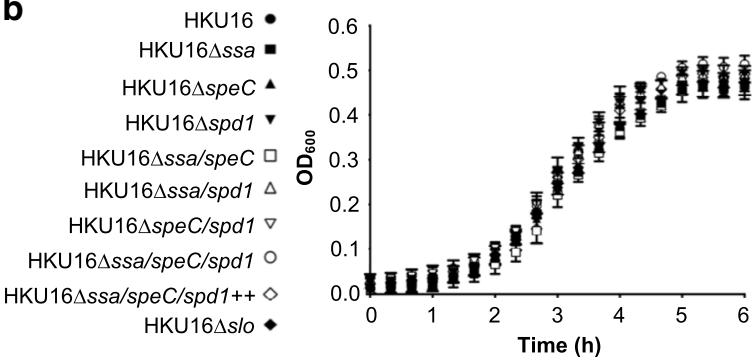

d

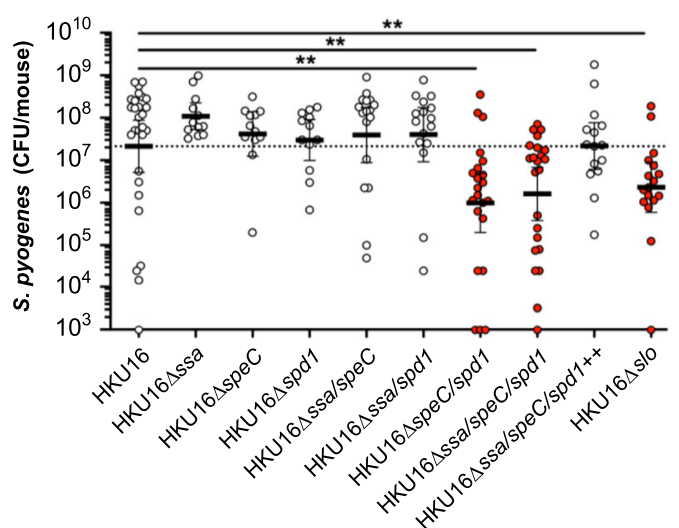

Fig. 4 ФHKU.vir-encoded exotoxins and SLO are critical for HKU16 nasopharyngeal infection. a Illustration of the genetic in-frame deletions of $\Phi H K U$. vir-encoded exotoxins in HKU16 as described in Materials and Methods. b Growth curves of indicated HKU16 strains in CDM from three independent experiments. Data are presented as mean values \pm SD. c Immunoblot detection of SSA, SpeC, Spd1, and SLO expression from indicated HKU16 strains. The molecular mass of each protein $(\mathrm{kDa})$ is indicated to the right. $\mathbf{d}$ Individual "humanized" B6 mice that express HLA-DR4, HLA-DQ8 and CD4 were nasally inoculated with $\sim 1 \times 10^{8}$ bacterial colony forming units (CFU) with indicated HKU16 strains and nasopharyngeal CFUs were assessed at $48 \mathrm{~h}$ post infection. Each symbol represents CFUs from an individual mouse $(n \geq 12)$. Presented is the geometric mean with $95 \%$ confidence interval. Significance was assessed using the Kruskal-Wallis test with the Dunn's multiple comparisons post-hoc test against the HKU16 control group ${ }^{* \star} p=0.0012$ for HKU16 $\Delta$ speC/spd1, ${ }^{\star \star} p=0.0041$ for HKU16 $\Delta s s a / s p e C / s p d 1$, and ${ }^{\star \star} p=0.01$ for HKU16 $\left.\Delta s / 0\right)$. Source data are provided as a Source Data file.

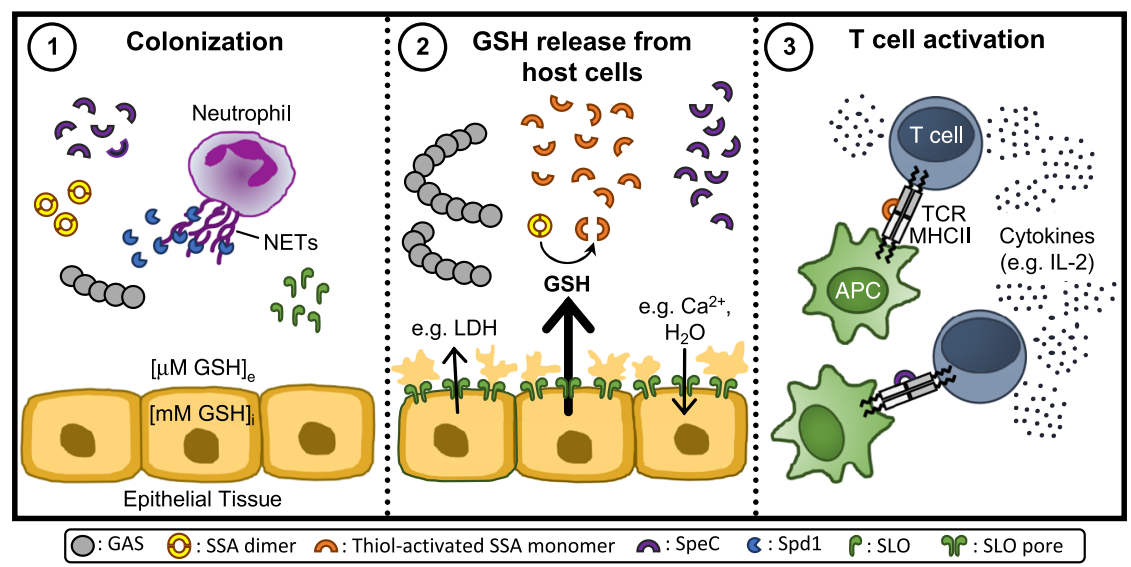

Fig. 5 Proposed mechanistic model outlining inter-relationships between SLO mediated cytotoxicity towards epithelial cells and SSA superantigen potency. (1) During initial bacterial colonization, GAS secretes the DNase Spd1 to escape neutrophil clearance, allowing GAS to establish infection. (2) As infection progresses, SLO binds to host cell membranes and then oligomerizes to form large pores which induces the release of lactate dehydrogenase $(\mathrm{LDH})$ and GSH from perforated host cells as well as cation influx ${ }^{71,72}$. Glutathione exists at a much higher concentration in the intracellular compartment $\left(\mathrm{GSH}_{\mathrm{i}}\right)$ than the extracellular space $\left(\mathrm{GSH}_{\mathrm{e}}\right)(\sim 1000$-fold) causing a significant difference in redox potential across the plasma membrane of eukaryotic cells. This gradient makes the extracellular and intracellular areas, respectively, oxidative and reductive. GSH efflux from perforated cells serves as a stimulus for SSA release, reduces SSA dimers and activates SSA monomers. (3) Thiol-activated SSA, in conjunction with other superantigens like SpeC, then cross-links major histocompatibility complex II molecules on antigen-presenting cells (APCs) and the variable region of the $\beta$-chain of T-cell receptor (TCR) to induce an overwhelming $\mathrm{T}$-cell response with uncontrolled cytokine release. 
grown at $37^{\circ} \mathrm{C}$ on $5 \%$ horse blood agar or statically in Todd-Hewitt broth supplemented with $1 \%$ yeast extract (THY) or chemically defined medium (CDM; Gibco RPMI 1640 with L-glutamine and phenol red (Life Technologies; 11875-093) supplemented with $0.7 \%(\mathrm{w} / \mathrm{v})$ D-Glucose, $1 \%(\mathrm{v} / \mathrm{v})$ BME vitamins (Sigma; B6891), 0.15 $\mathrm{mM}$ nucleobases (adenine, guanine, and uracil), and $0.02 \mathrm{mM}$ HEPES, $\mathrm{pH}$ 7.4). To facilitate fluorescent microscopy experiments, GAS strains were transformed with GFP-expressing plasmid pLZ12Km2-P23R-TA:GFP (Supplementary Methods). Escherichia coli (E. coli) strains MC1061 or XL1-blue, and BL21 (DE3), were used for cloning and protein expression, respectively. E. coli was grown in Luria-Bertani medium (LB). Where required, spectinomycin was used at $100 \mu \mathrm{g} \mathrm{ml}^{-1}$ (both GAS and E. coli), ampicillin was used at $100 \mu \mathrm{g} \mathrm{ml}^{-1}$ (E. coli), and kanamycin was used at $50 \mu \mathrm{g} \mathrm{ml}^{-1}$ (E. coli). All bacterial strains and plasmids are listed in Supplementary Table 1. Isogenic HKU16 mutants were generated using a highly efficient plasmid (pLZts) for creating markerless isogenic mutants ${ }^{64}$. All PCR primer sequences are provided in Supplementary Table 1. All gene deletions were confirmed by DNA sequence analysis (Australian Equine Genome Research Centre, University of Queensland, Brisbane, Australia). To examine fitness of wild-type and mutant strains, GAS were firstly grown overnight on horse blood agar. GAS were then inoculated into CDM to an optical density at $600 \mathrm{~nm}\left(\mathrm{OD}_{600}\right)$ of 0.01 . Lateexponential phase GAS grown in $\mathrm{CDM}\left(\mathrm{OD}_{600}\right.$ of 0.4$)$ were resuspended in ATCC Eagle's Minimum Essential Medium (EMEM; ATCC302003) supplemented with $10 \%$ heat-inactivated fetal bovine serum (FBS). Bacteria were then inoculated into 96-well microtiter plates and the growth curves measured using the FLUOstar Omega Microplate Reader (BMG Labtech) at $37^{\circ} \mathrm{C}$.

Transcriptomic and quantitative gene expression analysis. Total RNA was routinely isolated from bacterial cells as follows. Two volumes of RNAprotect (Qiagen) was added to the cultures, and bacterial cells were collected by centrifugation at $5000 \times g$ for $25 \mathrm{~min}$ at $4^{\circ} \mathrm{C}$. The dry cell pellet was stored at $-80^{\circ} \mathrm{C}$ overnight. Total RNA was extracted using the RNeasy minikit (Qiagen) with an additional mechanical lysis step using lysing matrix B tubes (MP Biomedicals). RNA samples were treated with Turbo DNase (Ambion) to eliminate contaminating genomic DNA and quantified using a NanoDrop instrument (Thermo Scientific). One microgram of RNA was converted to cDNA using the SuperScript VILO cDNA synthesis kit (Invitrogen). Resulting cDNA libraries were used for downstream analyses. RNA sequencing samples were taken from bacterial cultures grown in THY to late-exponential growth phase $\left(\mathrm{OD}_{600}\right.$ of $\left.\sim 0.7-0.8\right)$. Erythromycin was used at a concentration of $2 \mu \mathrm{g} \mathrm{ml}^{-1}$. Mitomycin $\mathrm{C}$ was added to early-exponential cultures $\left(\mathrm{OD}_{600}\right.$ of 0.25$)$ at a concentration of $0.2 \mu \mathrm{g} \mathrm{ml}^{-1}$. RNAsequencing was performed from Ribo-zero (rRNA depleted) Illumina libraries on a single Illumina HiSeq 2500 lane using v4 chemistry from 75 base pair paired-end reads. Reads were mapped to the HKU16 reference genome (alternatively termed HKU QMH11M0907901, GenBank accession number NZ_AFRY01000001) with BWA MEM (version 0.7.16). Relative read counts (per gene) and differential gene expression was determined using DESeq2 (v. 1.26.0) ${ }^{65}$ in R (v. 3.6.0). Genes with less than ten reads across all conditions and samples were removed. $P$ values were calculated using Wald test and adjusted for multiple testing using Benjamini-Hochberg/false-discovery rate. Read counts were visualized using the Integrative Genomics Viewer (IGV) and volcano plots were constructed using ggplot2 (v.3.2.1). To quantify gene expression, total RNA was isolated from bacterial cells harvested at late-exponential growth phase $\left(\mathrm{OD}_{600}\right.$ of 0.4$)$ in $\mathrm{CDM}$ grown in the presence or absence of $2 \mathrm{mM}$ redox-active compounds (L-Cysteine (Cys), DTT, reduced GSH, oxidized GSH (GSSG), and tris(2-carboxyethyl)phosphine (TCEP). Reverse transcription-PCR (RT-PCR) was performed using the primers specified in Supplementary Table 1, using SYBR green master mix (Applied Biosystems) according to the manufacturer's instructions. All data were analyzed using QuantStudio Real-Time PCR software v1.1 (QuantStudio 6 Flex, Life Technologies). Relative gene expression was calculated using the threshold cycle $\left(2^{-\Delta \Delta C T}\right)$ method with gyrA as the reference housekeeping gene ${ }^{66}$. All reactions were performed in triplicate from three independently isolated RNA samples.

\section{Purification of antigens and polyclonal antiserum production. The gene} encoding for the DNase Spd1, including nucleotides encoding the predicted signal peptide, was PCR amplified from genomic DNA of HKU16 and cloned into NdeI and HindIII sites of pET-28a. Point mutation of the active site residue Asn145 (Asn145Ala) ${ }^{67}$ was introduced using the QuikChange II site-directed mutagenesis kit (Agilent) to inactivate the Spd1 DNase (see Supplementary Table 1 for primer sequences). Wildtype Spd1 and inactivated Spd1 were produced by $0.5 \mathrm{mM}$ isopropyl $\beta$-D-1-thiogalactopyranoside (IPTG)-induced expression in E. coli BL21 (DE3), purified via nickel affinity chromatography, and $\mathrm{His}_{6}$ tags cleaved with $\mathrm{His}_{6}$-tagged tobacco etch virus (TEV) protease. The expression plasmids for wildtype SLO (pET-15b-SLO) ${ }^{49}$ and inactivated SLO carrying P427L and W535A mutations (pET-15b-SLOmut) ${ }^{68}$ were used to produce recombinant protein in $E$. coli BL21(DE3) following the same procedure as for Spd1. Recombinant proteins were analyzed for purity on $12 \%$ separating sodium dodecyl sulfate polyacrylamide gel electrophoresis (SDS-PAGE). Inactivated Spd1 and SLO were used to raise antisera in mice. Briefly, 4 - to 6 -week-old $\mathrm{BALB} / \mathrm{c}$ mice $(n=10)$ were immunized subcutaneously on days $0,14,21$, and 28 with $30 \mu \mathrm{g}$ of total protein adjuvanted with alum (Alhydrogel [2\%]; Brenntag) at a 1:1 ratio. One week following the last injection, mice were sacrificed and serum was collected for antibody titer analysis using ELISA.

Detection of exotoxins in GAS supernatants. Bacteria were routinely grown to late-exponential growth phase in CDM or THY where indicated. Filter-sterilized culture supernatants were precipitated with $10 \%$ trichloroacetic acid (TCA). TCA precipitates were resuspended in loading buffer (normalized to $\mathrm{OD}_{600}$ ) in the presence or absence of $100 \mathrm{mM}$ DTT. Samples were boiled for $10 \mathrm{~min}$, subjected to SDS-PAGE, and then transferred to polyvinylidene difluoride membranes for detection of immuno-reactive bands using a LI-COR Odyssey Imaging System (LICOR Biosciences). The primary antibodies used for the detection of SpeC, SSA and SpeB protein in GAS culture supernatants were rabbit antibody to SpeC (PCI333, Toxin Technology; 1:1000 dilution), affinity-purified rabbit antibody to SSA (produced by Mimotopes; 1:500 dilution) ${ }^{15}$ and affinity-purified rabbit antibody to SpeB (PBI222, Toxin Technology; 1:1,000 dilution). The murine primary antibody dilutions used for the detection of Spd1 and SLO were 1:1000 and 1:2000, respectively. Anti-rabbit IgG $(\mathrm{H}+\mathrm{L})$ (DyLight ${ }^{\mathrm{m}} 800$ 4X PEG Conjugate, NEB, 5151P) or anti-mouse IgG $(\mathrm{H}+\mathrm{L})$ (DyLight" $8004 \times$ PEG Conjugate, NEB, 5257S) were used as the secondary antibodies $(1: 10,000)$.

Recombinant superantigen purification. The SSA gene, lacking nucleotides encoding the predicted signal peptide, was PCR amplified from the $S$. pyogenes HKU16 chromosome using primers listed in Supplementary Table 1 and cloned into the $\mathrm{NcoI}$ and $\mathrm{BamHI}$ sites of a modified pET-41a protein expression vector that encodes an engineered TEV protease site to remove purification tags ${ }^{69}$. The C26S mutation was introduced into the ssa gene as above using primers listed in Supplementary Table 1 . Cloning of SpeC into the pET-41a vector was carried out as previously described ${ }^{20}$. Briefly, the speC gene lacking the coding sequence for the signal peptide was PCR amplified with primers NcoI_pET-41a_speC_F and BamHI_pET-41a_speC_R (Supplementary Table 1) and cloned into the $\mathbf{N} c o$ I and BamHI sites of pET-41a. Expression of the recombinant SSA and SpeC proteins was induced with $0.2 \mathrm{mM}$ IPTG in E. coli BL21(DE3) and purified as described above.

Superantigen activity as assessed by T-cell activation assay. Human periph eral blood mononuclear cells (PBMCs) isolated from freshly drawn heparinized venous blood from a healthy adult volunteer were resuspended in complete RPMI (cRPMI; RPMI1640, 10\% FBS, $0.1 \mathrm{mM}$ minimal essential media nonessential amino acids, $2 \mathrm{mM}$ L-glutamine, $1 \mathrm{mM}$ sodium pyruvate, $100 \mathrm{U} \mathrm{ml}^{-1}$ penicillin, $100 \mu \mathrm{g} \mathrm{ml}^{-1}$ streptomycin) and seeded at 200,000 cells per well in a 96-well plate. Sterile-filtered GSH dissolved in cRPMI (final concentration of $2 \mathrm{mM}$ ), or cRPMI alone, were added to each well $30 \mathrm{~min}$ prior to the addition of 10 -fold serial dilutions of recombinant superantigens. Cells were incubated at $37^{\circ} \mathrm{C}$ in $5 \% \mathrm{CO}_{2}$ for $18 \mathrm{~h}$. Spent cell culture supernatant was harvested and analyzed for human IL-2 by ELISA according to the manufacturer's instructions (eBiosciences).

Ex vivo whole blood model. Freshly drawn heparinized venous blood from a healthy adult volunteer was aliquoted $(180 \mu \mathrm{l})$ into wells of a 96-well plate. To validate hemolytic activity of SLO, increasing concentrations of recombinant wildtype SLO were added to give a final volume of $200 \mu \mathrm{l}$ per well and incubated at $37^{\circ} \mathrm{C}$ for $2 \mathrm{~h}$ with $5 \% \mathrm{CO}_{2}$. For bacterial infections, GAS strains were grown to lateexponential growth phase in $\mathrm{CDM}\left(\mathrm{OD}_{600}\right.$ of 0.4$)$, resuspended in Hanks Balanced Salt Solution (HBSS) at $\sim 1 \times 10^{8} \mathrm{CFU} \mathrm{ml}^{-1}$, and then added to whole blood to give a final volume of $200 \mu \mathrm{l}\left(\sim 2 \times 10^{6} \mathrm{CFU}\right)$. Growth of GAS strains was assessed $2 \mathrm{~h}$ post infection by plating serial dilutions for enumeration of CFUs. Plasma samples for detection of hemolysis and GSH release were obtained $4 \mathrm{~h}$ post-infection by centrifugation at $4800 \times g$ for $15 \mathrm{~min}$ at $4^{\circ} \mathrm{C}$. Controls included for each experiment were whole blood treated with HBSS (mock), or blood lyzed with $0.1 \%$ Triton $\mathrm{X}-100$.

Co-culture of $\boldsymbol{S}$. pyogenes with human pharyngeal cells. Human nasopharyngeal carcinoma epithelial cells Detroit 562 (ATCC CCL-138, Lot 70004014) were cultured at $37^{\circ} \mathrm{C}$ under a $5 \% \mathrm{CO}_{2}, 20 \% \mathrm{O}_{2}$ atmosphere in EMEM supplemented with $10 \%$ FBS in tissue culture vessels (Greiner Bio-one). At $90 \%$ confluency, cells were trypsinized and handled according to manufacturer's instructions. Detroit 562 cells were utilized for experiments at passage 8 and seeded at a density of $\sim 1.2 \times 10^{5}$ viable cells per well in 24 -well tissue culture plates, or $\sim 2.5 \times 10^{5}$ viable cells per well in 12-well plates $24 \mathrm{~h}$ prior to infection to allow the formation of confluent monolayers. Cells were grown at $37^{\circ} \mathrm{C}$ under $5 \% \mathrm{CO}_{2}$ until they formed a confluent monolayer. Immediately prior to infection, the cell culture medium was removed, and replaced with fresh medium. Increasing concentrations of recombinant wild-type SLO were added to cell monolayers and incubated for $2 \mathrm{~h}$. GAS strains were grown to late-exponential growth phase in $\mathrm{CDM}\left(\mathrm{OD}_{600}\right.$ of 0.4$)$ resuspended in cell culture medium, and then added to cell monolayers at a multiplicity of infection of 20. Controls included for each experiment were cells not exposed to bacteria or SLO (mock), or cells lyzed with $0.2 \%$ Triton X-100. At $2 \mathrm{~h}$ post-infection, plates were centrifuged at $500 \times g$ for $5 \mathrm{~min}$, then media was aspirated and stored at $-80^{\circ} \mathrm{C}$ until further processing. 
Cell membrane damage and GSH release assessment. SLO-induced hemolysis in whole blood was determined after collecting plasma samples and diluting 1:10 in phosphate-buffered saline (PBS). The amount of hemoglobin was measured spectrophotometrically at $405 \mathrm{~nm}$. Pharyngeal cell membrane disruption was quantified by measuring lactate dehydrogenase (LDH) release from cell supernatants, using CytoTox96 Non-Radioactive Cytotoxicity Assay (Promega; G1781), as per the manufacturer's instructions. Sample absorbance was measured spectrophotometrically at $490 \mathrm{~nm}$. GSH release was measured using the GSH-Glo GSH assay (Promega; V6912), as per the manufacturer's instructions, with the modification of mixing undiluted samples 1:1 with $2 \mathrm{mM}$ TCEP in wells of a white 96well plate (Greiner Bio-one) prior to use. Luminescent intensity of each sample was measured using a FLUOstar Omega Microplate Reader (BMG Labtech). Sample readings were analyzed by Prism 8 software and divided by the positive control for cell lysis to give a percentage of total hemolysis and cell membrane damage (LDH) for each sample.

Neutrophil killing assay. Human neutrophils were isolated from fresh heparinized whole blood using PolymorphPrep density gradient centrifugation (Axis-Shield) as per manufacturer's instructions. Following neutrophil harvest, hypotonic lysis was performed to remove residual erythrocytes. Purified neutrophils were infected with GAS at a multiplicity of infection of $0.1\left(1 \times 10^{6} \mathrm{cells} \mathrm{m}^{-1}\right.$ neutrophils: $1 \times 10^{5}$ bacterial $\mathrm{CFU} \mathrm{ml}{ }^{-1}$ ), centrifuged for $5 \mathrm{~min}$ at $370 \times g$ to synchronize phagocytosis, and then incubated for $30 \mathrm{~min}$ at $37^{\circ} \mathrm{C}$ under $5 \% \mathrm{CO}_{2}$. Control wells contained bacteria only. Infected neutrophils were then lysed using $0.025 \%$ Triton X-100 and serially diluted in sterile Milli-Q water, then plated on THY agar. Following overnight incubation at $37^{\circ} \mathrm{C}$, bacterial survival was calculated as the average total number of CFUs following incubation in the presence of neutrophils divided by CFUs in control wells.

NETs degradation assay. Freshly isolated purified human neutrophils were seeded on $12 \mathrm{~mm}$ Poly-D-lysine-coated $(0.01 \%$ solution overnight; Sigma-Aldrich; P7405) coverslips at a concentration of $1 \times 10^{6}$ cells ml $^{-1}\left(5 \times 10^{5}\right.$ cells ml $^{-1}$ per well) in a 24-well tissue culture plate. Neutrophils were stimulated with $25 \mathrm{nM}$ phorbol 12-myristate 13-acetate (PMA) (Cayman Chemical; 10008014), centrifuged for $5 \mathrm{~min}$ at $370 \times \mathrm{g}$, and incubated for $3 \mathrm{~h}$ at $37^{\circ} \mathrm{C}$ under $5 \% \mathrm{CO}_{2}$ to induce NET formation. Cell culture media was then removed, and the PMAstimulated neutrophils were infected with fluorescent GAS strains diluted in RPMI media containing $2 \%$ heat inactivated autologous human plasma and $5 \mathrm{mM} \mathrm{MgCl}_{2}$ at a multiplicity of infection of $10\left(1 \times 10^{7}\right.$ bacterial CFU ml ${ }^{-1}: 1 \times 10^{6} \mathrm{cells} \mathrm{ml}^{-1}$ PMN). Infected plates were centrifuged at $370 \times g$ for 5 min to promote cell interaction and then incubated for an additional $30 \mathrm{~min}$ at $37^{\circ} \mathrm{C}$ under $5 \% \mathrm{CO}_{2}$ Bovine pancreas DNase I at $5 \mu \mathrm{g} \mathrm{ml}^{-1}$ (Sigma; D5025) was used as a positive control to confirm NET degradation, while wells containing medium was used to confirm the formation of NETs. Cells were washed once with PBS, followed by fixation with $4 \%$ paraformaldehyde for $15 \mathrm{~min}$ at room temperature. After two washes, cells were incubated with $1 \mathrm{mM}$ SYTOX Orange Nucleic Acid Stain (Molecular Probes; S11368) for $15 \mathrm{~min}$ in the dark at room temperature to stain for NETs. After washing in 5\% (v/v) PBS, coverslips were embedded in Fluorescent Mounting medium (Dako; S30230) on microscopic glass slides and dried overnight in the dark at room temperature. Slides were stored at $4{ }^{\circ} \mathrm{C}$ until images were acquired. Samples were recorded using a Leica TCS SP8 Lightning confocal laser scanning microscope (Leica Microsystems) with a $63 \times$ oil immersion objective. GFP and SYTOX Orange were excited with 488 and $561 \mathrm{~nm}$ lasers, respectively, with images captured using sequential scanning. For each sample, a minimum of five randomly selected images per independent experiment performed in duplicate were acquired. For figure production, images were processed using ImageJ software (version 1.8.0) and the Enhance Local Contrast function was used to improve images for better visualization. For quantification of NET DNA degradation, the cell imaging analysis software CellProfiler (version 3.1.9) was employed. The percentage area of NETs per image was calculated as the area of neutrophil nuclei subtracted from the total area stained with SYTOX Orange. Images used for NET quantification were unenhanced.

HLA-B6 murine nasopharyngeal colonization model. For nasopharyngeal infection ${ }^{20,21}$, sex- and age-matched (9- to 13-week-old) transgenic C57BL/6J mice expressing human major histocompatibility complex II molecules DR4/DQ8 and human CD4 (HLA-B6) ${ }^{48}$ were infected with $\sim 1 \times 10^{8} \mathrm{CFU}$ per $15 \mu$ using $7.5 \mu \mathrm{l}$ to inoculate each nostril under methoxyflurane inhalation anesthetic. For infection, bacteria were cultured to late-exponential growth phase $\left(\mathrm{OD}_{600}\right.$ of 0.4$)$ in $\mathrm{CDM}$ supplemented with $2 \mathrm{mM}$ of L-Cys, washed and concentrated in CDM. Shamtreated mice only received CDM. Mice were sacrificed $48 \mathrm{~h}$ post infection, and the combined nasal turbinates, including the nasal associated lymphoid tissue and nasal turbinates, were removed. Tissue was homogenized in HBSS in lysing matrix F tubes (MP Biomedicals), serially diluted, and plated on horse blood agar for enumeration of beta-hemolytic CFUs with a detection limit of $10^{3} \mathrm{CFU}$ per mouse.

Statistical analysis. All statistical analysis was completed using Prism software (GraphPad; version 8.4.3). Significance was calculated using, where indicated, the two-tailed unpaired Student's $t$ test, one-way analysis of variance with Dunnett's multiple comparisons post-hoc test, and the Kruskal-Wallis test with the Dunn's multiple comparisons post hoc test. A $p$ value less than 0.05 was determined to be statistically significant.

Ethics statement. The human ethics protocol for the isolation of human blood from healthy volunteers for use in T-cell activation assays was approved by the Health Sciences Research Ethics Board at Western University (Ontario, Canada) (Protocol \#110859). Human blood donation for use in whole blood proliferation assays, neutrophil killing assays and NET degradation assays were conducted in accordance with the Australian National statement on ethical conduct in human research ${ }^{70}$, in compliance with the regulations governing experimentation on humans, and was approved by the University of Queensland medical research ethics committee (2010001586) and the University of Wollongong Human Research Ethics Committee (HE08/250). Informed consent was obtained from all participants. Animal experiments were performed according to the Australian code of practice for the care and use of animals for scientific purposes. Permission was obtained from the University of Queensland ethics committee to undertake this work (SCMB/140/16/NHMRC). Animal holding rooms were held at $22^{\circ} \mathrm{C}$ (with a range of $20-26^{\circ} \mathrm{C}$ ). Humidity was kept between $\sim 50$ and $70 \%$. A $12 \mathrm{~h} \mathrm{light/dark}$ cycle $(6 \mathrm{am}-6 \mathrm{pm})$ was used. Temperature and light cycle were both monitored. Humidity and temperature was recorded daily by animal holding room technicians

Reporting summary. Further information on research design is available in the Nature Research Reporting Summary linked to this article.

\section{Data availability}

Source data are provided with this paper. The HKU16 reference genome (alternatively termed HKU QMH11M0907901) is available on NCBI under the GenBank accession number NZ_AFRY01000001. Illumina read data are available on NCBI under the sample accession numbers relating to the three conditions (in triplicate): THY (ERS1091539, ERS1091548, and ERS1091557); THY plus erythromycin (ERS1091542, ERS1091551, and ERS1091560); THY plus mitomycin C (ERS1091545, ERS1091554, and ERS1091563).

Received: 21 December 2019; Accepted: 1 September 2020; Published online: 06 October 2020

\section{References}

1. Katz, A. R. \& Morens, D. M. Severe streptococcal infections in historical perspective. Clin. Infect. Dis. 14, 298-307 (1992).

2. Morens, D. M., Folkers, G. K. \& Fauci, A. S. The challenge of emerging and reemerging infectious diseases. Nature 430, 242-249 (2004).

3. Luk, E. Y. et al. Scarlet fever epidemic, Hong Kong, 2011. Emerg. Infect. Dis 18, 1658-1661 (2012).

4. Tse, H. et al. Molecular characterization of the 2011 Hong Kong scarlet fever outbreak. J. Infect. Dis. 206, 341-351 (2012).

5. Turner, C. E. et al. Scarlet fever upsurge in England and molecular-genetic analysis in North-West London, 2014. Emerg. Infect. Dis. 22, 1075-1078 (2016).

6. Chalker, V. et al. Genome analysis following a national increase in scarlet fever in England 2014. BMC Genomics 18, 224 (2017).

7. Park, D. W. et al. Incidence and characteristics of scarlet fever, South Korea, 2008-2015. Emerg. Infect. Dis. 23, 658-661 (2017).

8. Liu, Y. et al. Resurgence of scarlet fever in China: a 13-year population-based surveillance study. Lancet Infect. Dis. 18, 903-912 (2018).

9. Lamagni, T. et al. Resurgence of scarlet fever in England, 2014-16: a population-based surveillance study. Lancet Infect. Dis. 18, 180-187 (2018).

10. Lynskey, N. N. et al. Emergence of dominant toxigenic M1T1 Streptococcus pyogenes clone during increased scarlet fever activity in England: a populationbased molecular epidemiological study. Lancet Infect. Dis. 19, 1209-1218 (2019).

11. Yung, C. F. \& Thoon, K. C. A 12 year outbreak of scarlet fever in Singapore. Lancet Infect. Dis. 18, 942 (2018)

12. Walker, M. J. et al. Detection of epidemic scarlet fever group A Streptococcus in Australia. Clin. Infect. Dis. 69, 1232-1234 (2019).

13. Demczuk, W., Martin, I., Domingo, F. R., MacDonald, D. \& Mulvey, M. R. Identification of Streptococcus pyogenes M1UK clone in Canada. Lancet Infect. Dis. 19, 1284-1285 (2019).

14. Walker, M. J. \& Brouwer, S. Scarlet fever makes a comeback. Lancet Infect. Dis. 18, 128-129 (2018).

15. Davies, M. R. et al. Emergence of scarlet fever Streptococcus pyogenes emm12 clones in Hong Kong is associated with toxin acquisition and multidrug resistance. Nat. Genet. 47, 84-87 (2015).

16. Ben Zakour, N. L. et al. Transfer of scarlet fever-associated elements into the group A Streptococcus M1T1 clone. Sci. Rep. 5, 15877 (2015). 
17. You, Y. et al. Scarlet fever epidemic in China caused by Streptococcus pyogenes serotype M12: epidemiologic and molecular analysis. EBioMedicine 28, 128-135 (2018).

18. Silva-Costa, C., Carrico, J. A., Ramirez, M. \& Melo-Cristino, J. Scarlet fever is caused by a limited number of Streptococcus pyogenes lineages and is associated with the exotoxin genes ssa, speA and speC. Pediatr. Infect. Dis. J. 33, 306-310 (2014).

19. Proft, T. \& Fraser, J. D. In Streptococcus pyogenes: Basic Biology to Clinical Manifestations (eds Ferretti, J. J., Stevens, D. L. \& Fischetti, V. A.) 445-486 (Univ. Oklahoma Health Sciences Center, 2016).

20. Kasper, K. J. et al. Bacterial superantigens promote acute nasopharyngeal infection by Streptococcus pyogenes in a human MHC Class II-dependent manner. PLoS Pathog. 10, e1004155 (2014).

21. Zeppa, J. J. et al. Nasopharyngeal infection by Streptococcus pyogenes requires superantigen-responsive Vbeta-specific T cells. Proc. Natl Acad. Sci. USA 114, 10226-10231 (2017)

22. Fossum, G. H., Lindbaek, M., Gjelstad, S., Dalen, I. \& Kvaerner, K. J. Are children carrying the burden of broad-spectrum antibiotics in general practice? Prescription pattern for paediatric outpatients with respiratory tract infections in Norway. BMJ Open 3, e002285 (2013).

23. Bisno, A. L. Acute pharyngitis. N. Engl. J. Med. 344, 205-211 (2001).

24. Min, Y. H., Kwon, A. R., Yoon, E. J., Shim, M. J. \& Choi, E. C. Translational attenuation and mRNA stabilization as mechanisms of erm(B) induction by erythromycin. Antimicrob. Agents Chemother. 52, 1782-1789 (2008).

25. Banks, D. J., Lei, B. \& Musser, J. M. Prophage induction and expression of prophage-encoded virulence factors in group A Streptococcus serotype M3 strain MGAS315. Infect. Immun. 71, 7079-7086 (2003).

26. Morita, A., Imada, Y., Igarashi, H. \& Yutsudo, T. Serologic evidence that streptococcal superantigens are not involved in the pathogenesis of Kawasaki disease. Microbiol Immunol. 41, 895-900 (1997).

27. Norrby-Teglund, A. et al. Plasma from patients with severe invasive group A streptococcal infections treated with normal polyspecific IgG inhibits streptococcal superantigen-induced $\mathrm{T}$ cell proliferation and cytokine production. J. Immunol. 156, 3057-3064 (1996).

28. Schrage, B., Duan, G., Yang, L. P., Fraser, J. D. \& Proft, T. Different preparations of intravenous immunoglobulin vary in their efficacy to neutralize streptococcal superantigens: implications for treatment of streptococcal toxic shock syndrome. Clin. Infect. Dis. 43, 743-746 (2006).

29. Broudy, T. B., Pancholi, V. \& Fischetti, V. A. Induction of lysogenic bacteriophage and phage-associated toxin from group A streptococci during coculture with human pharyngeal cells. Infect. Immun. 69, 1440-1443 (2001).

30. Broudy, T. B., Pancholi, V. \& Fischetti, V. A. The in vitro interaction of Streptococcus pyogenes with human pharyngeal cells induces a phage-encoded extracellular DNase. Infect. Immun. 70, 2805-2811 (2002).

31. Sundberg, E. J. et al. Structures of two streptococcal superantigens bound to TCR beta chains reveal diversity in the architecture of $\mathrm{T}$ cell signaling complexes. Structure 10, 687-699 (2002).

32. De Marzi, M. C. et al. Cloning, expression and interaction of human T-cell receptors with the bacterial superantigen SSA. Eur. J. Biochem. 271 4075-4083 (2004).

33. Proft, T., Arcus, V. L., Handley, V., Baker, E. N. \& Fraser, J. D. Immunological and biochemical characterization of streptococcal pyrogenic exotoxins I and J (SPE-I and SPE-J) from Streptococcus pyogenes. J. Immunol. 166, 6711-6719 (2001).

34. Li, P. L., Tiedemann, R. E., Moffat, S. L. \& Fraser, J. D. The superantigen streptococcal pyrogenic exotoxin C (SPE-C) exhibits a novel mode of action. J. Exp. Med. 186, 375-383 (1997).

35. Proft, T., Moffatt, S. L., Berkahn, C. J. \& Fraser, J. D. Identification and characterization of novel superantigens from Streptococcus pyogenes. J. Exp. Med. 189, 89-102 (1999).

36. Tweten, R. K. Cholesterol-dependent cytolysins, a family of versatile poreforming toxins. Infect. Immun. 73, 6199-6209 (2005).

37. Schafer, F. Q. \& Buettner, G. R. Redox environment of the cell as viewed through the redox state of the glutathione disulfide/glutathione couple. Free Radic. Biol. Med. 30, 1191-1212 (2001).

38. Alouf, J. E. Streptococcal toxins (streptolysin O, streptolysin S, erythrogenic toxin). Pharm. Ther. 11, 661-717 (1980).

39. Bessen, D. E. Tissue tropisms in group A Streptococcus: what virulence factors distinguish pharyngitis from impetigo strains? Curr. Opin. Infect. Dis. 29, 295-303 (2016).

40. Aziz, R. K. et al. Mosaic prophages with horizontally acquired genes account for the emergence and diversification of the globally disseminated M1T1 clone of Streptococcus pyogenes. J. Bacteriol. 187, 3311-3318 (2005).

41. Sumby, P. et al. Evolutionary origin and emergence of a highly successful clone of serotype M1 group A Streptococcus involved multiple horizontal gene transfer events. J. Infect. Dis. 192, 771-782 (2005).

42. Walker, M. J. et al. DNase Sda1 provides selection pressure for a switch to invasive group A streptococcal infection. Nat. Med. 13, 981-985 (2007).
43. Afshar, B. et al. Enhanced nasopharyngeal infection and shedding associated with an epidemic lineage of emm3 group A Streptococcus. Virulence 8, 1390-1400 (2017)

44. Brinkmann, V. et al. Neutrophil extracellular traps kill bacteria. Science 303, 1532-1535 (2004)

45. Buchanan, J. T. et al. DNase expression allows the pathogen group A Streptococcus to escape killing in neutrophil extracellular traps. Curr. Biol. 16, 396-400 (2006).

46. Park, H. S. et al. Primary induction of CD4 T cell responses in nasal associated lymphoid tissue during group A streptococcal infection. Eur. J. Immunol. 34, 2843-2853 (2004)

47. Wang, B. et al. Induction of TGF-beta1 and TGF-beta1-dependent predominant Th17 differentiation by group A streptococcal infection. Proc. Natl Acad. Sci. USA 107, 5937-5942 (2010).

48. Chen, Z. et al. Humanized transgenic mice expressing HLA DR4-DQ3 haplotype: reconstitution of phenotype and HLA-restricted T-cell responses. Tissue Antigens 68, 210-219 (2006).

49. Timmer, A. M. et al. Streptolysin O promotes group A Streptococcus immune evasion by accelerated macrophage apoptosis. J. Biol. Chem. 284, 862-871 (2009).

50. Uchiyama, S. et al. Streptolysin O rapidly impairs neutrophil oxidative burst and antibacterial responses to group A Streptococcus. Front. Immunol. 6, 581 (2015).

51. Zhu, L. et al. Contribution of secreted NADase and streptolysin $\mathrm{O}$ to the pathogenesis of epidemic serotype M1 Streptococcus pyogenes infections. Am. J. Pathol. 187, 605-613 (2017).

52. Hsieh, Y. C. \& Huang, Y. C. Scarlet fever outbreak in Hong Kong, 2011. J. Microbiol. Immunol. Infect. 44, 409-411 (2011).

53. Chen, M. et al. Outbreak of scarlet fever associated with emm12 type group A Streptococcus in 2011 in Shanghai, China. Pediatr. Infect. Dis. J. 31, e158-e162 (2012).

54. Lau, E. H., Nishiura, H., Cowling, B. J., Ip, D. K. \& Wu, J. T. Scarlet fever outbreak, Hong Kong, 2011. Emerg. Infect. Dis. 18, 1700-1702 (2012).

55. Yang, P. et al. Characteristics of group A Streptococcus strains circulating during scarlet fever epidemic, Beijing, China, 2011. Emerg. Infect. Dis. 19, 909-915 (2013).

56. Brouwer, S., Lacey, J. A., You, Y., Davies, M. R. \& Walker, M. J. Scarlet fever changes its spots. Lancet Infect. Dis. 19, 1154-1155 (2019).

57. Brosnahan, A. J., Mantz, M. J., Squier, C. A., Peterson, M. L. \& Schlievert, P. M. Cytolysins augment superantigen penetration of stratified mucosa. $J$. Immunol. 182, 2364-2373 (2009).

58. Veeravalli, K., Boyd, D., Iverson, B. L., Beckwith, J. \& Georgiou, G. Laboratory evolution of glutathione biosynthesis reveals natural compensatory pathways. Nat. Chem. Biol. 7, 101-105 (2011).

59. Brenot, A., King, K. Y., Janowiak, B., Griffith, O. \& Caparon, M. G. Contribution of glutathione peroxidase to the virulence of Streptococcus pyogenes. Infect. Immun. 72, 408-413 (2004).

60. Reniere, M. L. Reduce, induce, thrive: bacterial redox sensing during pathogenesis. J. Bacteriol. 200, e00128-18 (2018).

61. Ku, J. W. \& Gan, Y. H. Modulation of bacterial virulence and fitness by host glutathione. Curr. Opin. Microbiol. 47, 8-13 (2018).

62. Commons, R. et al. Superantigen genes in group A streptococcal isolates and their relationship with emm types. J. Med. Microbiol. 57, 1238-1246 (2008).

63. Chochua, S. et al. Emergent invasive group A Streptococcus dysgalactiae subsp. equisimilis, United States, 2015-2018. Emerg. Infect. Dis. 25, 1543-1547 (2019).

64. Barnett, T. C., Daw, J. N., Walker, M. J. \& Brouwer, S. In Group A Streptococcus: Methods and Protocols (eds Proft, T. \& Loh, J.) 59-69 (Springer, 2020).

65. Love, M. I., Huber, W. \& Anders, S. Moderated estimation of fold change and dispersion for RNA-seq data with DESeq2. Genome Biol. 15, 550 (2014).

66. Brouwer, S. et al. Endopeptidase PepO regulates the SpeB cysteine protease and is essential for the virulence of invasive M1T1 Streptococcus pyogenes. J. Bacteriol. 200, e00654-17 (2018).

67. Korczynska, J. E., Turkenburg, J. P. \& Taylor, E. J. The structural characterization of a prophage-encoded extracellular DNase from Streptococcus pyogenes. Nucleic Acids Res. 40, 928-938 (2012).

68. Rivera-Hernandez, T. et al. Differing efficacies of lead group A streptococcal vaccine candidates and full-length $M$ protein in cutaneous and invasive disease models. mBio 7, e00618-16 (2016).

69. Rahman, A. K. M. N. U. et al. Molecular basis of TCR selectivity, crossreactivity, and allelic discrimination by a bacterial superantigen: integrative functional and energetic mapping of the SpeC-V beta 2.1 molecular interface. J. Immunol. 177, 8595-8603 (2006).

70. Australian Government National Health and Medical Research Council. National Statement on Ethical Conduct In Human Research (NHMRC, 2015).

71. Palmer, M. The family of thiol-activated, cholesterol-binding cytolysins. Toxicon 39, 1681-1689 (2001). 
72. Cywes Bentley, C., Hakansson, A., Christianson, J. \& Wessels, M. R. Extracellular group A Streptococcus induces keratinocyte apoptosis by dysregulating calcium signalling. Cell Microbiol. 7, 945-955 (2005).

\section{Acknowledgements}

We kindly acknowledge James McCluskey (University of Melbourne, Melbourne, Australia) for providing HLA-B6 mice transgenic for human CD4 and HLA-DR4-DQ8 genes; Luke McAlary (University of Wollongong, Wollongong, Australia) for assistance with microscopy; and assistance from the sequencing and pathogen informatics core teams at The Wellcome Trust Sanger Institute (Hinxton, UK). We thank Thomas Proft, Mitchell Acev, Heema Vyas, and Jason McArthur for providing genetic constructs. This work was supported by grants from the National Health and Medical Research Council of Australia, the Illawarra Health and Medical Research Institute, the Sanming Project of Medicine in Shenzhen, China (SZSM201911014), The Wellcome Trust, UK, the National Institutes of Health, USA, and the Canadian Institutes of Health Research.

\section{Author contributions}

S.B., T.C.B., G.D., V.N., K.Y.Y., Y.Y., J.K.M., M.L.S.S., M.R.D., and M.J.W. conceived this study. S.B., T.C.B., D.L., K.J.K., B.L.S., G.D., V.N., K.Y.Y., Y.Y., J.K.M., M.L.S.S., M.R.D., and M.J.W. planned experiments. S.B., T.C.B., D.L., K.J.K., L.M., M.G.J., D.M.P.D.O.,

T.R.H., A.J.C., J.R., and M.R.D. performed the experiments. G.D., V.N.,. K.Y.Y., and J.K.M. provided essential reagents and strains. S.B. and M.J.W. wrote the paper and all authors reviewed and revised the paper.

\section{Competing interests}

The authors declare no competing interests.

\section{Additional information}

Supplementary information is available for this paper at https://doi.org/10.1038/s41467020-18700-5.

Correspondence and requests for materials should be addressed to M.J.W.

Peer review information Nature Communications thanks Sven Hammerschmid and Saija Kiljunen for their contribution to the peer review of this work.

Reprints and permission information is available at http://www.nature.com/reprints

Publisher's note Springer Nature remains neutral with regard to jurisdictional claims in published maps and institutional affiliations.

(c) (i) Open Access This article is licensed under a Creative Commons Attribution 4.0 International License, which permits use, sharing, adaptation, distribution and reproduction in any medium or format, as long as you give appropriate credit to the original author(s) and the source, provide a link to the Creative Commons license, and indicate if changes were made. The images or other third party material in this article are included in the article's Creative Commons license, unless indicated otherwise in a credit line to the material. If material is not included in the article's Creative Commons license and your intended use is not permitted by statutory regulation or exceeds the permitted use, you will need to obtain permission directly from the copyright holder. To view a copy of this license, visit http://creativecommons.org/ licenses/by/4.0/.

(C) The Author(s) 2020 